\title{
Transcription Factor Foxd1 Is Required for the Specification of the Temporal Retina in Mammals
}

\author{
Maria Isabel Carreres, ${ }^{1}$ Augusto Escalante, ${ }^{1 \star}$ Blanca Murillo, ${ }^{1 \star}$ Geraud Chauvin, ${ }^{1}$ Patricia Gaspar, ${ }^{2}$ Celia Vegar, ${ }^{1}$ \\ and Eloisa Herrera ${ }^{1}$ \\ ${ }^{1}$ Instituto de Neurociencias, Consejo Superior de Investigaciones Científicas y Universidad Miguel Hernández, Campus San Juan de Alicante, 03550 \\ Alicante, Spain, and 2Inserm, Unité Mixte de Recherche-S 839, Université Pierre et Marie Curie and Institut du Fer à Moulin, 75005 Paris, France
}

The organization of the visual system is different in birds and mammals. In both, retinal axons project topographically to the visual targets in the brain; but whereas in birds visual fibers from the entire retina decussate at the optic chiasm, in mammals, a number of axons from the temporal retina diverge at the midline to project ipsilaterally. Gain-of-function experiments in chick raised the hypothesis that the transcription factor Foxd1 specifies retinal temporal identity. However, it remains unknown whether Foxd1 is necessary for this function. In mammals, the crucial role of Foxd1 in the patterning of the optic chiasm region has complicated the interpretation of its cell-autonomous function in the retina. Furthermore, target molecules identified for Foxd1 are different in chicks and mice, leading to question the function of Foxd1 in mammals. Here we show that in the mouse, Foxd1 imprints temporal features in the retina such as axonal ipsilaterality and rostral targeting in collicular areas and that EphA6 is a Foxd1 downstream effector that sends temporal axons to the rostral colliculus. In addition, our data support a model in which the desensitization of EphA6 by ephrinA5 in cis is not necessary for the proper functioning of EphA6. Overall, these results indicate that Foxd 1 functions as a conserved determinant of temporal identity but reveal that the downstream effectors, and likely their mechanisms of action, are different in mammals and birds.

\section{Introduction}

The organization of the visual system in mammals and birds is significantly different. In both vertebrate classes, retinal axons project to their target tissues in a topographic manner; but in mammals, sensory inputs are also segregated into eye-specific domains in the primary visual targets, the dorsal lateral geniculate nucleus (dLGN) and the superior colliculus (SC). In mammals, corresponding points in the two retinas are brought together through partial decussation of the optic nerves at the level of the optic chiasm, whereas in the visual system of birds, all retinal axons decussate at the midline (for review, see Martin, 2009).

Nasal axons project to the caudal SC/tectum, whereas temporal axons go to rostral collicular/tectal areas. Foxd1 is the only transcription factor described, to date, as specifically expressed in the early developing temporal retina (Hatini et al., 1994). Ectopic expression of Foxd1 in the nasal chick retina is sufficient to send retinal fibers to the rostral tectum (Yuasa et al., 1996), which has

\footnotetext{
Received Jan. 24, 2011; revised; accepted Feb. 12, 2011.

Research in the laboratory of E.H. was supported by grants from the Spanish Ministry of Science and Innovation (MICINN; Grant BFU2007-61831) and the CONSOLIDER-Ingenio Program (CDS2007-023). A.E. holds an FPU fellowship, and M.I.C. and B.M. hold FPI fellowships from MICINN. We thank A. Barco and C. García-Frigola for critical reading of this manuscript. We are also grateful to G. López-Bendito's laboratory for helpful discussion. We thank $S$. Brown and L. Brown for generously sharing the Zic 2 antibody and R. Hindges for the EphA6 and EphA5 probes. We also thank E. Lai for the Foxd1 mice.

*A.E. and B.M. contributed equally to this work.

Correspondence should be addressed to Eloisa Herrera, Instituto de Neurociencias, Consejo Superior de Investigaciones Científicas y Universidad Miguel Hernández, Campus San Juan de Alicante, Av. Ramón y Cajal, s/n 03550 Alicante, Spain. E-mail: e.herrera@umh.es.

DOI:10.1523/JNEUROSCI.0394-11.2011

Copyright $\odot 2011$ the authors $\quad 0270-6474 / 11 / 315673-09 \$ 15.00 / 0$
}

promoted the idea that Foxd1 defines temporal identity in the retina. This gain-of-function experiment was performed in chicks, and so far, no loss-of-function assays have yet demonstrated the requirement of Foxd 1 in the establishment of topographic maps. In mice, the genetic removal of Foxd 1 compromises the survival at birth (Hatini et al., 1996), complicating further investigations about its contribution to the postnatal establishment of topographic maps. In addition, Foxd1 is implicated in the patterning of the future optic chiasm region, and although Foxd1-deficient embryos display aberrant axonal projections at the optic chiasm (Herrera et al., 2004), it is unclear whether this misrouting is a consequence of the absence of Foxd1 in the retina, in the chiasmatic region, or in both.

Strikingly, the distribution of EphAs and the molecules described as downstream of Foxd1 are not the same in the mouse and chick retinas (Takahashi et al., 2003, 2009; Herrera et al., 2004). In the chick retina, the ectopic expression of Foxd 1 in the optic cup induces the upregulation of EphA3 and the downregulation of ephrinA5 (Yuasa et al., 1996; Takahashi et al., 2003, 2009). EphAs and ephrinAs are tyrosine kinase membrane proteins involved in the establishment of topography in several sensory systems. Retinal temporal axons express high levels of EphAs, whereas caudal areas in the visual targets express high levels of ephrinAs. The binding (in trans) of retinal EphAs to tectal ephrinAs mediates a repulsive signal that forces axons to project rostrally (Cheng et al., 1995; Drescher et al., 1995; Feldheim et al., 2000). EphrinAs, in addition to being expressed in the targets, are also expressed in the retina (Marcus et al., 1996; Connor et al., 1998; Hornberger et al., 1999). In the chick retina, the substantial coexpression (in cis) of EphAs and ephrinAs in retinal 
ganglion cells (RGCs) has supported the idea that cis- and trans-presented ephrinAs exert opposing functions on EphA3, with retinal ephrinA5 acting as a modifier of the EphA-mediated signaling (Carvalho et al., 2006). This view is in agreement with recent biochemical evidence in vitro showing that EphA3 and ephrinA5 interact in cis (Monschau et al., 1997; Connor et al., 1998; Reber et al., 2004; Carvalho et al., 2006). In mice, however, EphA3 is not expressed in RGCs (Feldheim et al., 1998; Brown et al., 2000). Therefore, the function of Foxd 1 in the specification of temporal identity in mammals has been questioned.

To determine whether Foxd1 is not only sufficient but also necessary for the establishment of topographic maps and to clarify the function of Foxd1 and its downstream effectors in the development of the mammalian visual system, we ectopically transduced Foxd 1 in the embryonic mouse retina to analyze the phenotype of the retinal axons. Also, we observe alterations in retinotopy caused by the lack of Foxd 1 in the temporal retina and identify EphA6 as a Foxd1 downstream effector that sends axons to the rostral SC and the dorsocaudal dLGN. Our data also strongly suggest that ephrinA 5 in the mouse retina acts as a receptor rather than by affecting EphA6-mediated signaling.

\section{Materials and Methods}

Animals. We use Foxd $1{ }^{\text {tmLai }}$ mice that have been described previously (Hatini et al., 1996). In these mice, the coding region of Foxd1 is substituted for the lac $Z$ gene. These mice are Foxd1 null mice, and for simplicity, from now on we will refer to them as Foxd1 ${ }^{-1-}$. For coculture experiments, Foxd1 mice were crossed with transgenic Tg (CAG/Acr-EGFP) mice (Okabe et al., 1997). For the rest of the experiments, (DBA/2 $\times$ C57BL/6)F1 embryos or mice were obtained from a timed pregnancy breeding colony at the Instituto de Neurociencias (IN). In all cases, embryonic day 0 (E0) was defined as midnight of the night before a plug was found. Animal protocols were approved by the IN Animal Care and Use Committee and European and national laws.

DNA plasmids. The coding sequences of enhanced green fluorescent protein (EGFP) and Foxd1 were cloned into pCAG plasmids. The Foxd1 probe was cloned by PCR from the 3' untranslated region sequence (forward primer, 5'-GGGAGAGCGAAGGTAGGACT-3'; reverse primer, 5'-CTTTGAAAATTAAGCATTTTATTTCTG-3' ${ }^{\prime}$. The EphA6 and EphA5 probes are similar to those used by Brown et al. (2000). The EphrinA5 probe is similar to that used by Vanderhaeghen et al. (2000). A mutated form of EphA6, EphA6EE, was constructed as done by Egea et al. (2005). Two tyrosine residues, Y606 and Y612, equivalent to the EphA4 tyrosine residues Y596 and Y602, were replaced by glutamic acid. The coding sequences of EGFP, EphrinA5, EphA6, and EphA6EE were inserted in a plasmid to drive their expression under the CAG promoter.

In utero electroporation and axon quantification. In utero electroporation was performed as described by García-Frigola et al. (2007). To visualize optic chiasms from electroporated embryos, pregnant mothers were killed $4 \mathrm{~d}$ after electroporation, and embryos were removed. Then, embryonic brains were dissected out, and the optic chiasms were viewed en face in a whole mount with a fluorescence dissecting scope. The quantification of the uncrossed projection was performed as done by Herrera et al. (2003) and García-Frigola et al. (2008).

In situ hybridization, immunohistochemistry, and quantitative reverse transcription-PCR. In situ hybridization using digoxigenin-labeled riboprobes was performed on $20 \mu \mathrm{m}$ cryosections as described previously (Schaeren-Wiemers and Gerfin-Moser, 1993). The tissue was then processed for immunohistochemistry with anti-Zic2 antibodies (Brown et al., 2003) as done by Herrera et al. (2003), and the images were inverted using Adobe Photoshop. Quantitative reverse transcription-PCR (qRTPCR) was performed as described previously (García-Frigola et al., 2008). The primers used were as follows: FoxD1 forward (f), $5^{\prime}$-ACTCAGCCCTGGCCTTGC-3'; FoxD1 reverse (r), 5'-GCGGGAGGAGTGAGAATCTAGAA-3'; FoxG1 $1_{\mathrm{f}}$, 5'-GCCAGATTTCCATGTGTGCA-3'; FoxG1 $1_{\mathrm{r}}$, 5'-GCGTCCACCAGATAGCTCC-3'; Gapdh $_{\mathrm{f}}, 5^{\prime}$-CTTCACCACCATGGAGAAGGC-3'; Gapdh $_{\mathrm{r}}, 5^{\prime}$-CATGGACTGTGGTCATGAGCC-3'; EphrinA5 f, 5'-TGTGGCCTTTCCCCAATTC-3'; EphrinA5 ${ }_{\mathrm{r}}$, 5'-GAGCAAGGAGCAGAACGTG-3'; EphA 5 , $5^{\prime}$-TGAATGCGTCGAGCAGAGTG-3'; EphA5 ${ }_{r}, \quad$ 5'-CCAAAGAACCATGTTCTGCCA-3'; EphA6 $_{\text {f }}$, 5'-AAGGGCTGGGTTTTTAAATGG-3'; EphA6 ${ }_{r}$, 5'-GAGAGCCTCCTGCAGTCACTG-3'. Transcript levels were calculated using the comparative $\mathrm{Ct}$ method normalized to glyceraldehyde-3-phosphate dehydrogenase mRNA levels. The final results were expressed relative to wild-type retinas using the $2^{-(\Delta \Delta \mathrm{Ct})} \pm$ SEM formula. 

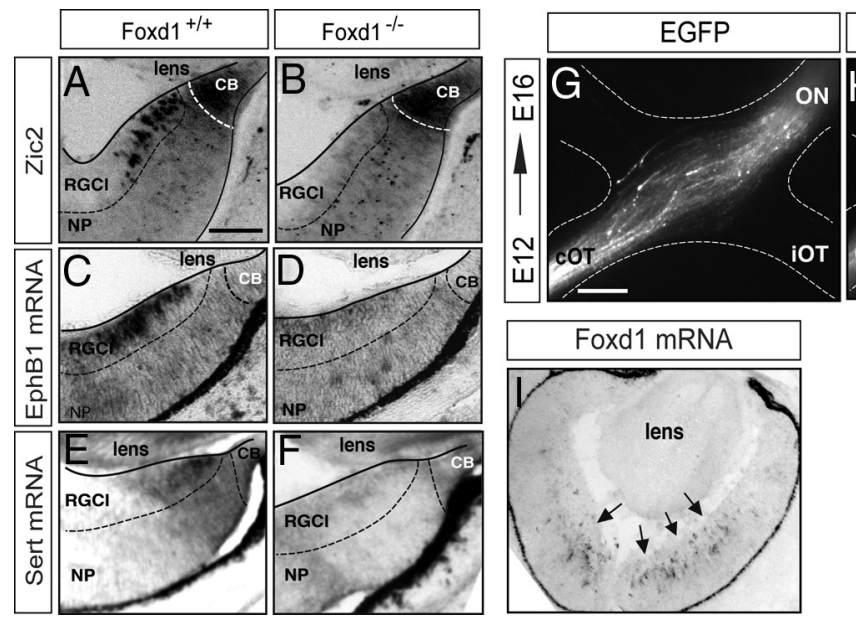

Figure 2. Foxd1 nourishes iRGC identity. $\boldsymbol{A}, \boldsymbol{B}$, Immunohistochemistry against Zic2 in E16 retinal sections from the VT region of wild-type and littermate Foxd1 null embryos shows that although Zic2 expression is normal in the ciliary body cells, it is not detected in differentiated iRGCs in Foxd1-deficient retinas (Foxd1 ${ }^{-/-}$). $\mathbf{C}-\boldsymbol{F}$, In situ hybridization for EphB1 $(\boldsymbol{C}, \boldsymbol{D})$ or Sert $(\boldsymbol{E}, \boldsymbol{F})$ in retinal sections from wild-type and Foxd $1^{-1-}$ mice shows that the expression of these two molecules is highly downregulated in VT retina in the absence of Foxd1. NP, Neural progenitors; RGCl, retinal ganglion cells layer; CB, ciliary body. Scale bar, $200 \mu \mathrm{m}$. G, $\boldsymbol{H}$, Images show the chiasm region of E16 embryos electroporated with EGFP or Foxd1/EGFP at E12. 0N, Optic nerve; i0T, ipsilateral optic tract; $\mathrm{COT}$, contralateral optic tract. The white arrow in $\boldsymbol{H}$ points to the ectopic ipsilateral projection. The arrowhead points to misrouted axons in the contralateral optic nerve. Scale bar, $200 \mu \mathrm{m}$. I, In situ hybridization to Foxd 1 in a retinal section from an E16 embryo electroporated with Foxd1/EGFP confirms ectopic expression of Foxd1 in the central retina (arrows).
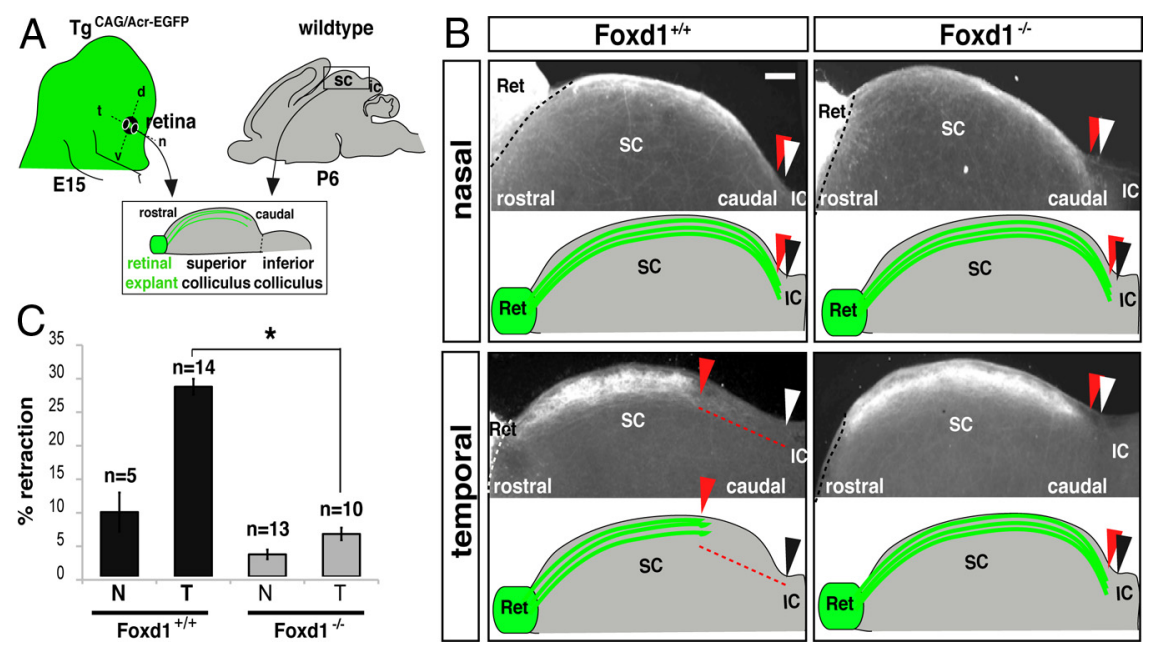

Figure 3. Temporal axons from Foxd1 null embryos project aberrantly to caudal areas in the SC. $A$, Schema representing the experimental procedure in which EGFP-labeled retinal explants from wild-type or Foxd1 mutant mice are confronted with wild-type collicular slices. $\boldsymbol{B}$, Cocultures of nasal or temporal retinal explants from E14 Foxd ${ }^{+/+} ; \mathrm{Tg}{ }^{\mathrm{CAG} / \mathrm{Acr}-\mathrm{EGFP}}$ or Foxd1 ${ }^{-1-} ; \mathrm{Tg}^{\text {CAG/Acr-EGFP }}$ embryos confronted to collicular sagittal slices from P6 wild-type mice. Retinal axons from nasal regions of Foxd1 ${ }^{-1-} ; \mathrm{Tg}^{\text {CAG/Acr-EGFP }}$ mice or from Foxd1 ${ }^{+1+} ; \mathrm{Tg}^{\text {CAG/Acr-EGFP }}$ reach the end of the colliculus. Temporal axons from Foxd1 ${ }^{+/+} ; \mathrm{Tg}{ }^{\text {CAG/Acr-EGFP }}$ mice project to mediorostral collicular positions, whereas temporal axons from Foxd1 ${ }^{\text {lacZ/lacZ; }}$ Tg ${ }^{\text {CAG/Acr-EGFP }}$ project to caudal areas. White arrowheads mark the end of the SC, and gray arrowheads indicate the location where most of retinal axons stop their growth. Ret, Retinal explant; IC, inferior colliculus. Red dashed lines highlight the long distance between the white and the gray arrowhead in the control situation. Scale bar, $100 \mu \mathrm{m}$. $\boldsymbol{C}$, Quantification of the coculture experiments shows that the aberrant behavior of temporal axons in the absence of Foxd1 is significantly different from wild-type temporal axons. T, temporal; N, Nasal. The values represent means \pm SEM (error bars) of the number of cocultures indicated in each case $\left({ }^{*} p<0.05\right.$, Student's unpaired $t$ test).

Cocultures. Coculture experiments were performed as done by Nicol et al. (2006). Briefly, retinal explants from the temporal and nasal retina were dissected from E14-E15 mouse embryos and were grown as organotypic cocultures confronted with parasagittal slices of the SC from wild-type mice at postnatal day 6 (P6). After $12 \mathrm{~d}$ in vitro, coculture preparations were fixed, and retinal axons were visualized by immunostaining to green fluorescent protein (1:1000; AVES Laboratories). Reti-

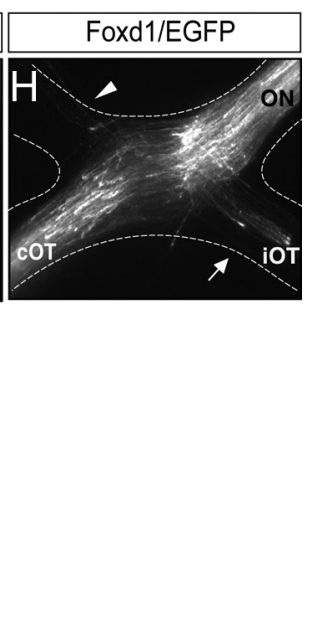

nal explants cocultured with collicular sagittal slices were photographed in a conventional epifluorescence microscope. For each slice, the length of the collicular surface containing EGFP axons was measured and considered as the total length. Then, the length of the collicular surface empty of EGFP axons was measured, starting at the most caudal tip of the SC; this measure was considered as the length of retraction. The percentage of retraction was expressed as a ratio of the total length divided by the length of retraction.

$S C$ and $d L G N$ analysis. Newborn animals were anesthetized and perfused with $4 \%$ paraformaldehyde (PFA). Brains were removed, and telencephalon was dissected to expose the entire dLGN and SC before photographing them. Then, brains were postfixed overnight in $4 \%$ PFA, embedded in 3\% agarose, and sagittaly or coronally sectioned. Next, $100 \mu \mathrm{m} \mathrm{sec}-$ tions of the dLGN and SC were photographed with a conventional epifluorescence microscope.

\section{Results}

\section{Foxd1 prepatterns ventrotemporal} identity in the mammalian retina During the development of the mouse optic chiasm, early-generated RGC axons originating from the dorsal-central retina reach the developing ventral diencephalon at E12 and grow across the ventral midline to establish the correct position of the X-shaped optic chiasm. A number of these early axons, instead of crossing the midline, project into the ipsilateral side of the brain, forming a transient ipsilateral projection. These early stages of the optic chiasm formation are similar in both mammals and birds (Guillery, 1982; O'Leary et al., 1983; Mason and Sretavan, 1997). Later, around E14, a subpopulation of RGCs in the peripheral ventrotemporal (VT) retina of mammals, but not in birds, develops a new ipsilateral projection that is maintained throughout adult life (O'Leary et al., 1983; Guillery et al., 1995).

The transcription factor Foxd1 is expressed in the mouse temporal retina at E10 (Hatini et al., 1994) and in a more restricted VT retinal quadrant at E14 and E16 (Herrera et al., 2004). To obtain a precise localization at intermediate ages, we performed in situ hybridization for Foxd 1 mRNA in retinal sections from E11-E16 embryos. Foxd1 mRNA was detected at E11-E12 in retinal progenitors of the temporal retina (Fig. 1A). Over time, expression is downregulated (Fig. $1 A$ ) and shuts down by E17 (data not shown). An accumulation of Foxd1 mRNA was also observed in the peripheral VT retina from E12 to E14 (Fig. 1A).

The transcription factor Zic2 is expressed postmitotically in the peripheral VT retina and specifies the permanent ipsilaterally projecting RGCs (iRGCs) (Herrera et al., 2003). Zic2 is also expressed in the ciliary body (CB) (Herrera et al., 2003), where it 

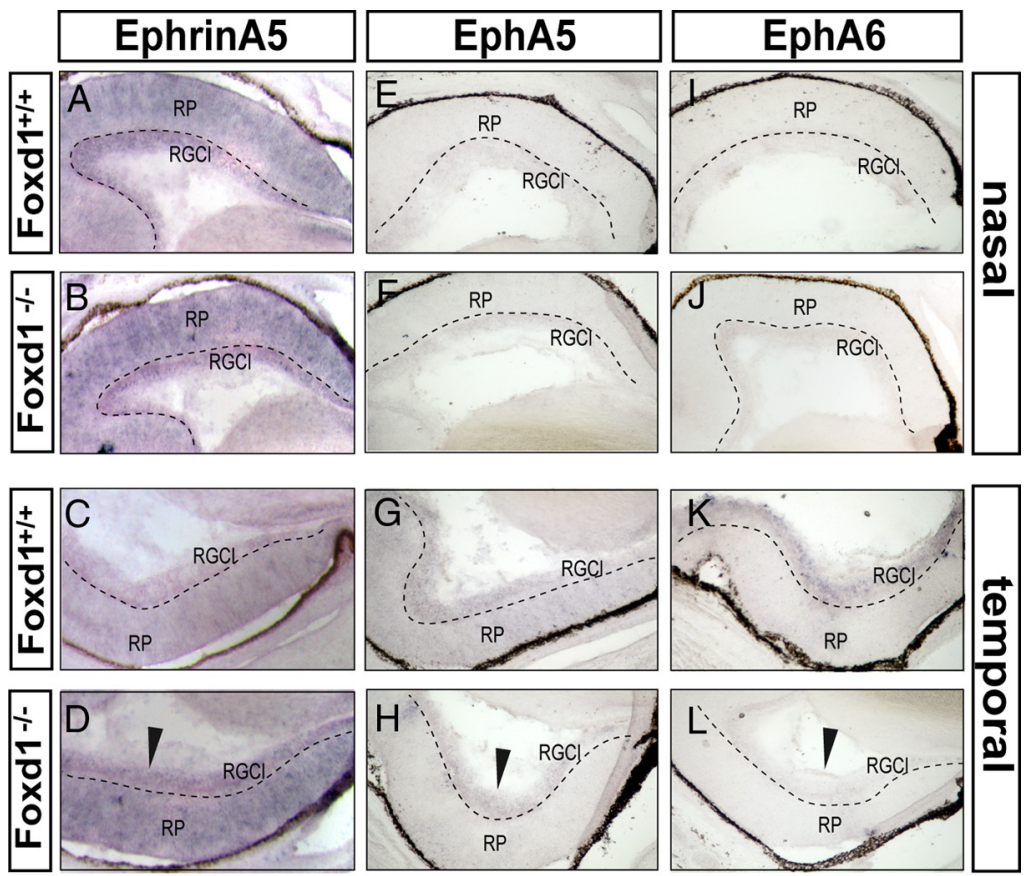

M
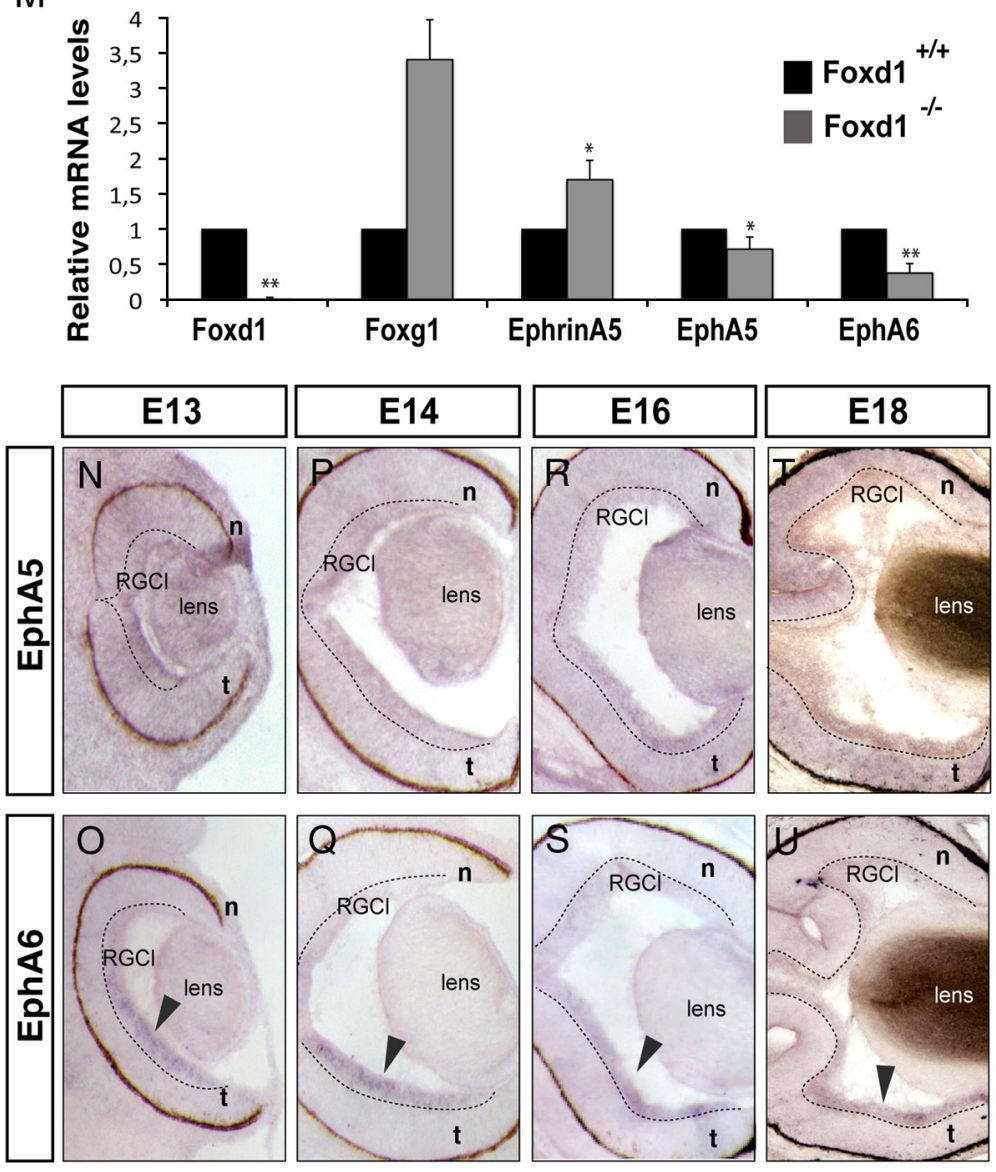

Figure 4. Expression of EphAs and ephrinAs in wild-type and Foxd1 mutant retinas. $\boldsymbol{A}-\boldsymbol{L}$, Images show in situ hybridization using specific probes for ephrinA5, EphA5, and EphA6 in horizontal retinal sections from E17 wild-type and Foxd1 ${ }^{-1}$ embryos. The expression of these molecules in the nasal retina of Foxd $1^{-1-}$ mice is unaltered. In contrast, arrows in $\boldsymbol{D}, \boldsymbol{H}$, and $\boldsymbol{L}$ highlight the altered expression of ephrinA5, EphA5, and EphA6 in the temporal retina of Foxd $1^{-1-}$ mice. $\boldsymbol{M}$, The graph shows the results from qRT-PCR assays comparing mRNA levels of Foxd1, EphA5, EphA6, Foxg1, and EphrinA5 in E16 retinas from Foxd1 ${ }^{+/+}$and Foxd1 ${ }^{-1-}$ embryos. In the absence of Foxd1, global levels of EphA5 and EphA6 mRNAs decrease, whereas ephrinA5 and Foxg1 mRNA levels increase. mRNA levels for each molecule are shown relative to the wild-type values. At least two retinas for each condition were pooled per experiment, and the average of seven experiments is shown. Error bars indicate $\pm \mathrm{SEM}\left({ }^{*} p<0.05\right.$ plays an undetermined function. Since $\mathrm{Zic} 2$ is one of the few proteins expressed in the peripheral VT retina, we used it to better characterize Foxd1 expression. In situ hybridization for Foxd1 mRNA was combined with Zic2 immunofluorescence. At E13, accumulation of Foxd1 mRNA was detected in a region located more centrally than the Zic2-positive cells of the CB. At E14, Zic2 expression in the CB decreased and became visible in recently differentiated iRGCs (Herrera et al., 2003). At this stage, Foxd1 mRNA filled the gap located between Zic2-positive iRGCs and the CB. At E15, Zic2 was strongly expressed in the iRGCs, but Foxd1 mRNA expression in the peripheral VT was very faint (Fig. 1B). This spatiotemporal expression pattern suggested that Foxd1 precedes the expression of Zic2. Foxd1 seemed to be expressed in progenitors leaving the cell cycle that will give rise to differentiated Zic2-positive RGCs. To confirm this hypothesis, we reexamined the retinal expression pattern of Zic2 in the absence of Foxd 1 and observed, as reported previously (Herrera et al., 2004), that Zic2 expression disappeared in the VT region of Foxd $1^{-1-}$ retinas. However, we also noted that Zic2 was maintained in the ciliary body cells, which never express Foxd1, indicating that Zic2 expression does not always depend on Foxd1 (Fig. $2 A, B)$.

We then analyzed the expression of two identified target genes of Zic2, the tyrosine kinase receptor EphB1 (GarcíaFrigola et al., 2008) and the serotonin transporter Sert (García-Frigola and Herrera, 2010). Previous observations showing that EphB1 mRNA was no longer expressed in the VT retina of Foxd1 ${ }^{-1-}$ mice were confirmed, and we also observed that Sert mRNA was strongly downregulated in the Foxd1 mutant mice (Fig. 2C-F).

These results suggested that Foxd 1 activates the program that specifies the identity of RGCs in the VT retinal segment. To test this possibility, plasmids bearing the coding sequence of EGFP alone or together with the coding sequence of Foxd1 (Foxd1/EGFP) were co-introduced into neurons of the center of the retina in E12E13 embryos by in utero electroporation

$\leftarrow$

${ }^{* *} p \leq 0.001$, Student's unpaired $t$ test). $\boldsymbol{N}-\boldsymbol{T}$, In situ hybridization for EphA5 in horizontal retinal sections from embryos at the indicated stages. $\mathbf{0}-\boldsymbol{U}$, In situ hybridization for EphA6 in horizontal retinal sections from embryos at the indicated stages. Arrows point to the strong expression of EphA6 in the temporal RGC layer (RGCl). 


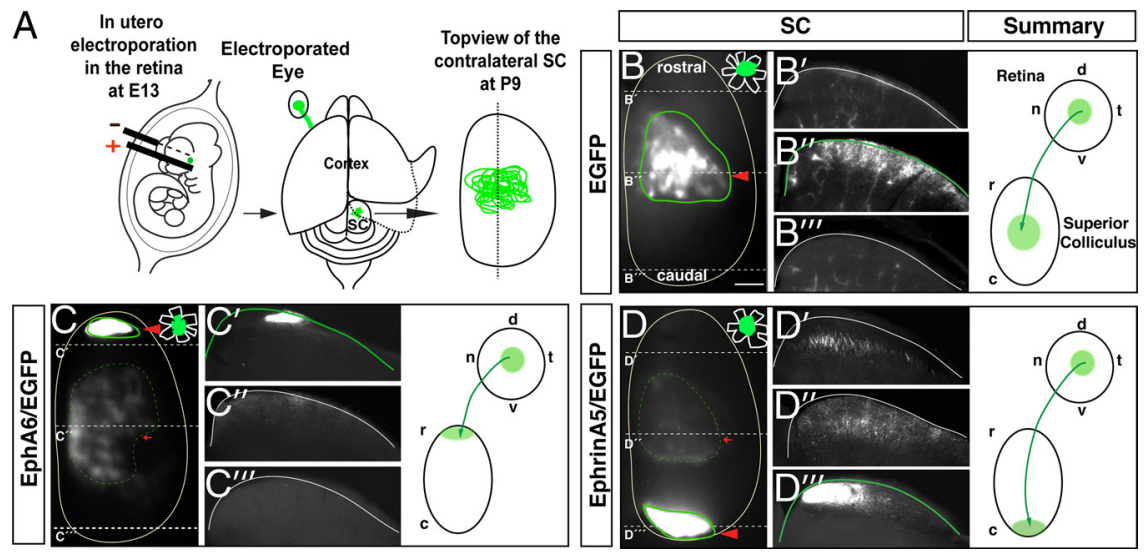

Figure 5. Ectopic expression of EphA6/EGFP or ephrinA5/EGFP sends retinal axons to the rostral and to the caudal colliculus, respectively. $\boldsymbol{A}$, Scheme summarizing the experimental procedure. Different combinations of plasmids were injected into the retinas of E13 embryos, and each embryo was then electroporated. Two weeks later, the cortex was removed, and the entire $S C$ was exposed for visualization of EGFP-expressing axons. $\boldsymbol{B}-\boldsymbol{D}$, The images show a top view of the entire SC of P9 mice electroporated at E13 with the plasmids indicated in each case. In the right corner of each panel, a drawing of the retina to indicate the location of targeted cells (green) is shown. The red arrowheads highlight the termination zone (TZ) of the majority of the targeted axons. Red arrows point the $\mathrm{TZ}$ of a minority of the targeted axons. Middle, Coronal sections at the levels indicated by dashed lines. Right, Schemes summarize the projection patterns of targeted RGCs. d, Dorsal; v, ventral; $t$, temporal; $n$, nasal; r, rostral; c, caudal. Scale bar, $200 \mu \mathrm{m}$.

[see García-Frigola et al. (2007) for electroporation methods]. Four days after electroporation, we analyzed the projection of targeted RGC axons at the optic chiasm. As expected, in embryos electroporated with EGFP alone, targeted axons crossed the midline and only very rarely entered the ipsilateral optic tract $(2.7 \pm$ $2.0 \%$ of the targeted axons in 7 of the 18 electroporated embryos) or the contralateral optic nerve $(3.2 \pm 1.8 \%$ of the targeted axons in 8 of the 18 of the electroporated embryos). In contrast, in embryos electroporated with Foxd1/EGFP, $13.40 \pm 2.5 \%$ of the targeted axons were misrouted to the ipsilateral tract in 29 of the 33 electroporated embryos $\left({ }^{*} p<0.05\right.$, Student's unpaired $t$ test $)$. The number of axons misrouted to the contralateral optic nerve also increased $(7.50 \pm 2.0 \%)$ in 22 of the 33 electroporated embryos $\left({ }^{*} p<0.05\right.$, Student's unpaired $t$ test $)$. This result indicates that the expression of Foxd 1 in the retina is sufficient to trigger the program that specifies ipsilaterality at the midline, demonstrating a function of this gene in the mouse retina that is independent of its previously reported role in the developing chiasmatic region (Herrera et al., 2004).

\section{Foxd1 is required for the correct targeting of temporal axons to rostral collicular areas}

In chicken, the ectopic expression of Foxd1 in the nasal retina causes mistargeting of RGC axons to the rostral instead of to the caudal tectum (Yuasa et al., 1996; Takahashi et al., 2009). However, whether Foxd1 is required for the establishment of retinotopy in the targets has not been determined by loss-of-function experiments. Foxd $1^{-1-}$ mice die at birth (Hatini et al., 1996), making it impossible to analyze the postnatal establishment of retinocollicular maps. To overcome this limitation, we used a retinocollicular coculture assay that recapitulates the topographic distribution of retinal axons in the SC (Nicol et al., 2006, 2007). Temporal or nasal retinal explants from E14-E15 wildtype or Foxd $1^{-/-}$mice were cocultured with collicular sagittal slices of P6 wild-type mice. To visualize RGC axons, Foxd1 ${ }^{+/-}$ mice were crossed with a transgenic mouse line that ubiquitously express EGFP under a potent general promoter (Tg CAG/Acr-EGFP) (Okabe et al., 1997) to generate FoxD ${ }^{+/+} ; \mathrm{Tg}^{\text {CAG/EGFP }}$ (control) and FoxD1 ${ }^{-1-}$;Tg CAG/EGFP (Foxd1null) retinal explants (Fig. $3 A$ ). After $12 \mathrm{~d}$ in culture, RGCs axons from control and Foxd1-null nasal explants projected to caudal regions of the colliculus, whereas control temporal retinal axons were found in more rostral collicular areas. In contrast, temporal RGC axons derived from explants of Foxd1null mice showed no topographic specificity and invaded the entire rostrocaudal extent of the SC (Fig. 3B,C). These experiments indicated that Foxd 1 is required for the correct targeting of temporal axons in rostral collicular areas.

\section{EphA6 and ephrinA5 are the Foxd1 downstream effectors that control topographic maps in mice}

In the chick retina, the ectopic expression of Foxd1 induces an upregulation of the axon guidance receptor EphA3 (Takahashi et al., 2009). However, EphA3 is not expressed in the mouse RGCs (Feldheim et al., 1998; Brown et al., 2000). Because the only two EphAs receptors expressed in a high-temporal to low-nasal gradient in the mouse retina are EphA5 and EphA6 (Feldheim et al., 2000), we investigated their expression levels in the absence of Foxd1, together with the expression of ephrinA5 that, according to previous studies, should be altered (Herrera et al., 2004). To this end, we performed in situ hybridization in horizontal retinal sections from E17 embryos. We did not observe differences between genotypes in the expression of EphA5, EphA6, or ephrinA5 in the nasal retinas. However, in the temporal retina, EphA5 and EphA6 mRNA expression was higher in wild-type embryos than in the Foxd $1^{-1-}$ littermates. As expected, ephrinA5 expression increased in the temporal retina of Foxd $1^{-l-}$ mice compared with wild-type mice (Fig. 4A-L).

To quantify these changes in the expression levels of EphA5/A6 and ephrinA5 in the Foxd1 mutants, we performed qRT-PCR assays on whole retinas from wild-type and Foxd1 ${ }^{-/-}$ littermate embryos. As a positive control, we examined the expression of Foxg1, which is upregulated in Foxd $1^{-1-}$ retinas (Herrera et al., 2004). Foxd1 mRNA expression was used as a negative control. These experiments confirmed the reduced expression of EphA5 and EphA6 and the increased levels of ephrinA5 in Foxd1 ${ }^{-/-}$compared with wild-type retinas (Fig. $4 M$ ). Then, we performed in situ hybridization for EphA6 and EphA5 in retinal sections of embryos from E12 to E18 and observed that EphA6 mRNA was highly and specifically expressed in temporal RGCs, whereas EphA5 mRNA expression was weaker (Fig. $4 \mathrm{~N}$ $U)$, confirming previous results (Reber et al., 2004) showing that EphA6 mRNA expression is more abundant than EphA5 mRNA in RGCs from the temporal retina.

EphA5 is important for the establishment of topographic maps in the mouse (Feldheim et al., 2004). However, although EphA6 expression in temporal RGCs seems to be higher than EphA5 expression, whether EphA6 controls the topographic mapping of mouse retinal axons to the rostral colliculus has not been addressed. Since central retina shows basal levels of EphA6 (Fig. 4), we expressed EphA6 ectopically in RGCs from the center of the retina by in utero electroporation to assess whether EphA6 is sufficient to control topographic mapping. We first determined by qRT-PCR the ectopic levels of EphA6 in our electroporation 
Table 1. Retinal-labeled axon projection phenotype at the SC and the dLGN after in utero electroporation

\begin{tabular}{|c|c|c|c|c|c|c|c|c|c|}
\hline \multirow[b]{2}{*}{ DNA injected } & \multirow[b]{2}{*}{ Age of electroporation } & \multirow[b]{2}{*}{ Age of analysis } & \multirow[b]{2}{*}{ Number of mice } & \multicolumn{3}{|l|}{ SC } & \multicolumn{3}{|c|}{$\mathrm{dLGN}$} \\
\hline & & & & Rostral & Central & Caudal & VR & Middle & $D C$ \\
\hline EGFP & E13 & P9 & 10 & - & $\uparrow$ & - & - & $\uparrow$ & - \\
\hline EphA6/EGFP & E13 & P9 & 8 & $\uparrow$ & $\downarrow$ & - & $\uparrow$ & $\downarrow$ & - \\
\hline EphrinA5/EGFP & E13 & P9 & 9 & - & $\downarrow$ & $\uparrow$ & - & $\downarrow$ & $\uparrow$ \\
\hline EphA6/ephrinA5/EGFP & E13 & P9 & 14 & 1 embryo showed $\downarrow$ & $\uparrow$ & 13 embryos showed $\downarrow$ & - & $\uparrow$ & - \\
\hline EphA6EE/EGFP & E13 & P9 & 12 & $\uparrow$ & $\downarrow$ & - & $\uparrow$ & $\downarrow$ & - \\
\hline EphA6EE/ephrinA5/EGFP & E13 & P9 & 9 & - & $\uparrow$ & 6 embryos showed $\downarrow$ & - & $\uparrow$ & - \\
\hline
\end{tabular}

$\uparrow$, High fluorescence intensity; $\downarrow$, low fluorescence intensity; —, no fluorescence; DC: dorsocaudal.

experiments. A $1.8 \pm 0.13$-fold increase $(n=9 ; p \leq 0.001$, Student's unpaired $t$ test) in the levels of EphA6 was observed. Then, to analyze the projection of targeted RGCs at the colliculus, embryos electroporated at E13 were postnatally killed at P9, when the establishment of visual maps is complete. Their colliculus were then sagittaly sectioned, and the projection pattern of targeted axons was analyzed. As expected, targeted axons from mice electroporated with EGFP alone $(n=10)$ always projected to central collicular areas. In embryos coelectroporated with EphA6 and EGFP plasmids (EphA6/EGFP; $n=$ 8 ), most of the projections were found in rostral positions (Fig. 5, Table 1). However, a small proportion of the targeted axons were detected in the central colliculus, the area that topographically corresponds to the targeted retinal region. As two different plasmids were coelectroporated to express EphA6 and EGFP, it is likely that those axons projecting correctly in the central SC correspond to the few RGCs that express EGFP but not EphA6 or those that express EphA6 at very low levels.

EphrinA5 is also significantly altered in the temporal retina in the absence of Foxd1. In chick, retroviral overexpression of ephrinA5 in the temporal retina causes temporal axons to elaborate axonal extensions and arborization in caudal positions instead of projecting in rostral positions in the tectum (Dütting et al., 1999). In mice, however, it is unknown whether the ectopic expression of ephrinA5 in the retina is sufficient to alter topographic mapping. To investigate whether this is the case, we ectopically expressed ephrinA5 by in utero electroporation in central RGCs. Ectopic expression of ephrinA5 in the central retina after electroporation was increased $2.5 \pm 0.2$ fold ( $n=9 ; p \leq 0.001$, Student's unpaired $t$ test) compared with the endogenous levels of expression. In P9 pups electroporated at E13 with ephrinA5/EGFP, the targeted axons were mostly found in caudal collicular regions in all the cases, although a minority of axons continued projecting to the central colliculus (Figs. 5; Table 1). As for EphA6, these few centrally projecting axons are likely those that express only EGFP but not ephrinA5.
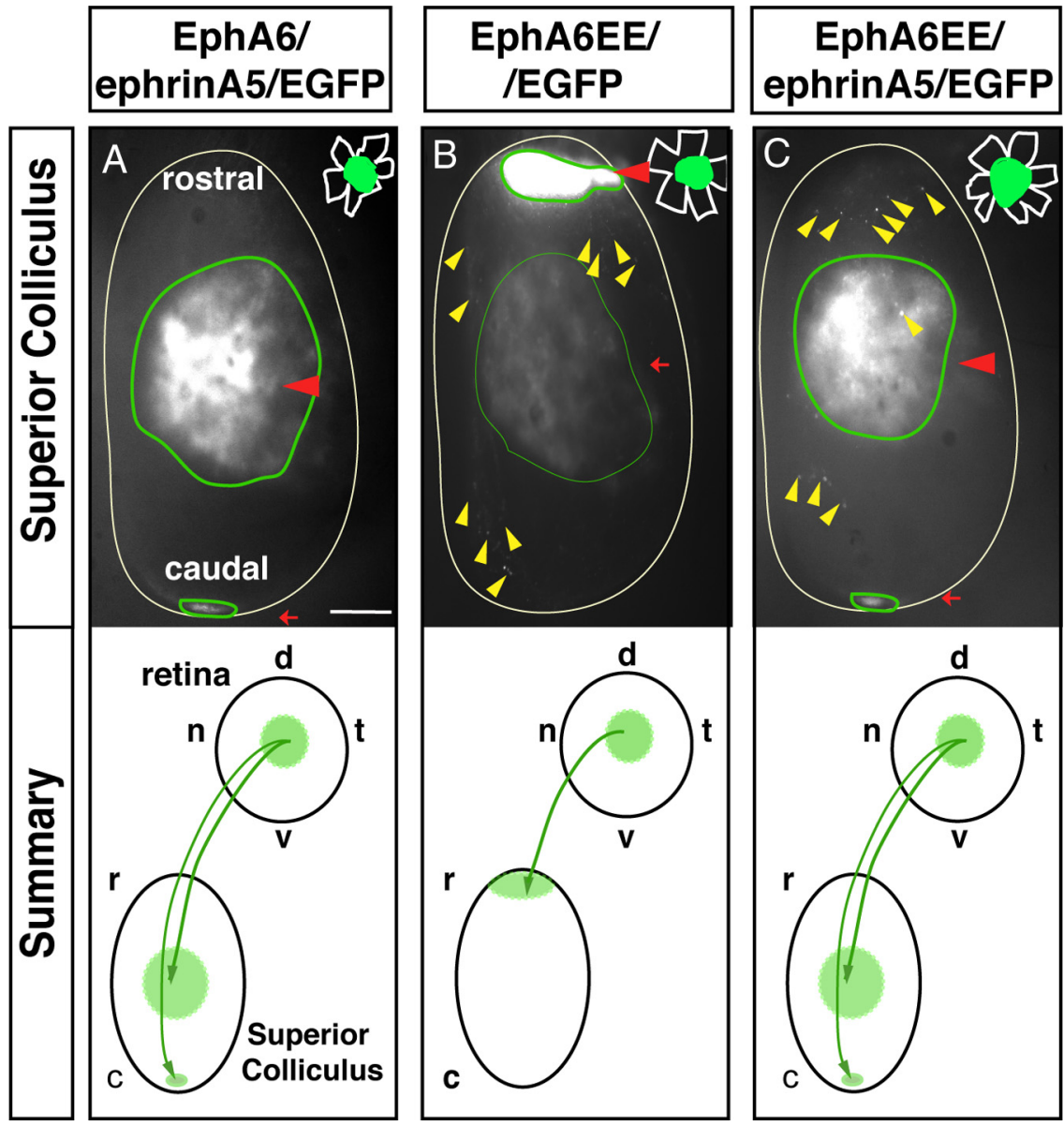

Figure 6. EphrinA5 acts as a receptor in the retina rather than a modifier of the EphA signaling. The images show top views of the SC of P9 mice electroporated at E13 with EphA6/ephrinA5/EGFP (A), EphA6EE/EGFP (B), or EphA6EE/ephrinA5/EGFP (C). Red arrowheads highlight the termination zone (TZ) of the majority of the targeted axons. Red arrows indicate the TZ of a minority of targeted axons. Yellow arrowheads point to axons that seem to be degenerating. In the right corner of each panel, a drawing of the retina to indicate the location of targeted cells (green) is shown. Below each picture, schemes summarize the projection patterns of targeted RGCs in the SC. d, Dorsal; v, ventral; t, temporal; n, nasal; r, rostral; c, caudal. Scale bar, $200 \mu \mathrm{m}$.

These results show that the ectopic expression of EphA6 or ephrinA5 in the mouse retina is sufficient to send axons to the rostral and caudal areas, respectively, in the SC.

EphrinA5 in the retina acts as a receptor rather than as a desensitizer of EphA6-mediated signaling

Two different mechanisms have been proposed for the action of ephrinAs in the retina. Pioneer theories suggested that ephrinAs could act as receptors mediating reverse signaling. However, the fact that they do not have an intracellular domain, together with biochemical and in vitro experiments in chick, raised the idea that they could rather be modulators of EphA-mediated signaling 

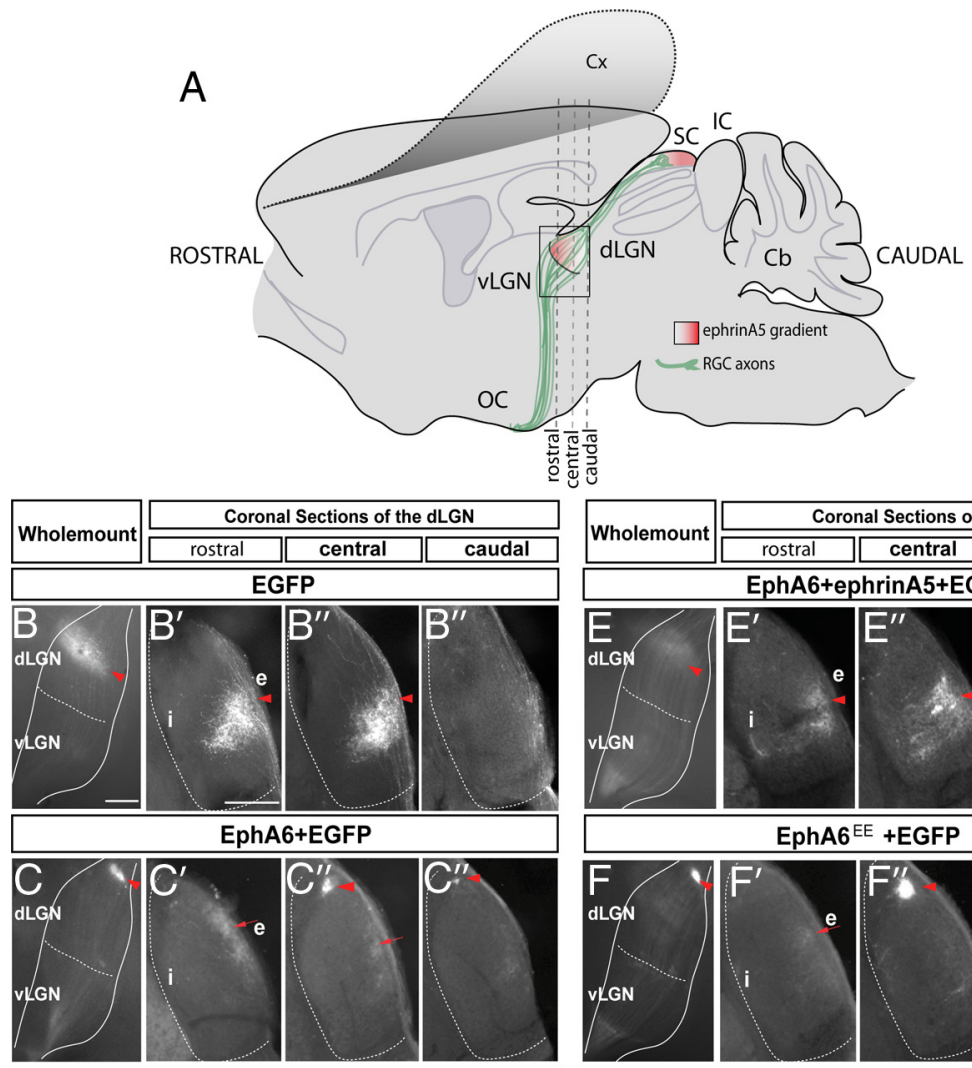

EphrinA5+EGFP
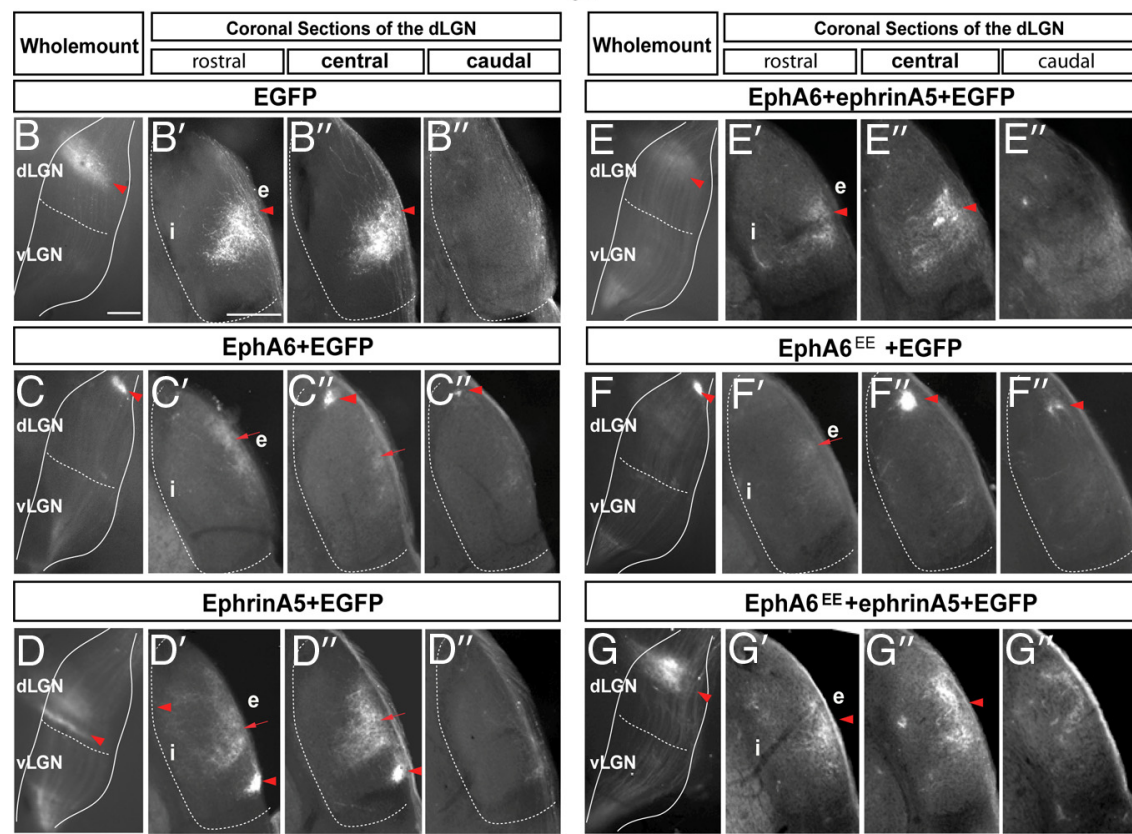

EphA6+ephrinA5+EGFP

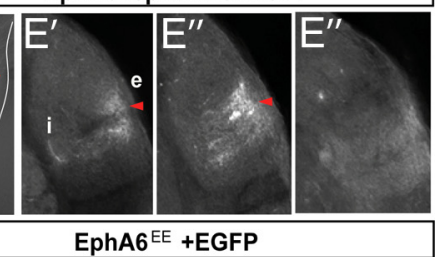

EphA6 $^{\mathrm{EE}}+\mathrm{EGFP}$

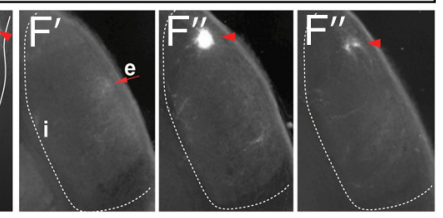

EphA6EE+ephrinA5+EGFP
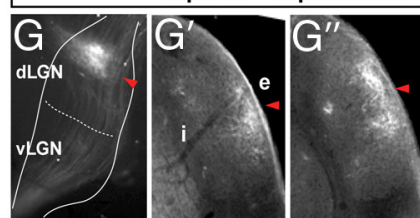

Figure 7. The EphA/ephrinA mechanisms that control the retinothalamic projection are similar than those controlling the retinocollicular projection. $A$, Schematic representation of a lateral view of the brain in which, after cortex removal, it is possible to observe electroporated retinal axons (green) at the LGN level (squared area). The gradient of ephrinA5 in the dLGN and SC is represented in red. $\boldsymbol{B}-\mathbf{G}$, Lateral view of the entire $L G N$ of $P 9$ mice that were electroporated at $E 13$ with the plasmids indicated in each case. At the right of the images depicting the entire LGNs, coronal sections of the rostral, central, and caudal regions of each $\mathrm{dLGN}$ are shown. Red arrowheads highlight the termination zone (TZ) of the majority of the targeted axons. Red arrows indicate the TZ of a minority of the targeted axons. Cx, Cortex; SC superior colliculus; IC, inferior colliculus; vLGN, ventral lateral geniculate nucleus; $\mathrm{dLGN}$, dorsal geniculate nucleus; $\mathrm{Cb}$, cerebellum; $O C$, optic chiasm; $i$, internal region of the $\mathrm{dLGN}$; e, external region of the dLGN. Scale bar, $200 \mu \mathrm{m}$.

(Carvalho et al., 2006). Recent studies have taken up again the idea that ephrinAs could function as receptors because although ephrinAs lack an intracellular domain, they might transduce reverse signaling through binding to p75 neurotrophin or/and through TrkB (Rashid et al., 2005; Marler et al., 2008). Despite these theories, the role of ephrinAs in the establishment of retinotopic maps is still an open question. To shed light on this issue, we coexpressed EphA6 and ephrinA5 together with EGFP in central RGCs, using single EGFP electroporation as controls. In $92 \%$ of the EphA6/ephrinA5/EGFP electroporated mice $(n=14)$, axons were found in central or caudal areas. In $8 \%$ of the cases, all the axons projected to the central areas, and in one case, we found the majority of the axons in the central collicular areas with a few axons in both caudal and rostral regions (Fig. 6A, Table 1). These results suggest that, when electroporated at the same concentrations, the activities of EphA6 and ephrinA5 tend to annul each other. We then electroporated a constitutively active version of EphA6 (EphA6EE). EphA6EE was constructed as other constitu-

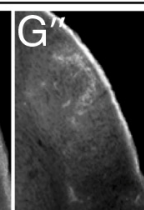

tively active forms for the tyrosine kinase receptor family (Chen et al., 1997; Pasini et al., 1997; Bardelli et al., 1998; Egea et al., 2005), where the two juxtamembrane tyrosines (Y596 and Y602) were replaced by two glutamic acid residues; therefore, this protein cannot be dephosphorylated and inactivated. In mice electroporated with EphA6EE/EGFP, a few targeted axons seemed to degenerate, but the bulk of the axons looked healthy and projected to rostral collicular areas in all cases $(n=12)$ (Fig. 6B). All mice coelectroporated with EphA6EE and ephrinA5 (EphA6EE/ephrinA5/EGFP) $(n=9)$, showed axons projecting in central positions in the SC (Fig. $6 C$ ), and in $66 \%$ of the cases, the axons went to both central and caudal areas (see Table 1 for a summary). As EphA6EE cannot be inactivated, these results support the hypothesis that ephrinA5 may trigger downstream signaling as a receptor independently of EphA6 phosphorylation. This signaling compensates, and in some cases overcomes, EphA6-mediated signaling, forcing axons to project into central or caudal areas.

\section{EphA6/ephrinA5 control topographic mapping in the dLGN by similar mechanisms than in the colliculus} We finally asked whether the targeting of retinothalamic axons was also specified by Foxd 1 and defined by guidance from its downstream effectors, EphA6 and ephrinA5. EphrinA5 is expressed in the dLGN in a high-rostral to low-caudal pattern (Feldheim et al., 1998), and EphA/ephrinA signaling mediates topography in the thalamus (Huberman et al., 2005; Pfeiffenberger et al., 2005). However, as in the case of the SC, the mechanisms of action of EphA/ephrinA signaling in the dLGN are unclear.

We sectioned the dLGNs of P9 mice that had been electroporated at E13 with EGFP alone, EphA6/EGFP, EphA6EE/EGFP, ephrinA5/EGFP, EphA6/eprhinA5/EGFP, or EphA6EE/ephrinA5/EGFP. As for the analysis of the SC, only mice electroporated in the central retina were considered in these experiments. In all the mice electroporated with EphA6 $(n=8)$, targeted axons coming from the central retina projected to the most dorsocaudal area in the dLGN. In the dLGN of mice electroporated with EphA6EE/EGFP $(n=12)$, we found a few degenerating axons as in the SC, but the majority of the targeted fibers projected to the dorsocaudal area. Mice electroporated with ephrinA5 $(n=9)$ showed axons projecting to ventrorostral (VR) areas in all the cases. After electroporation with EphA6 or with ephrinA5 plasmids, we found weak fluorescence in the central portion of the dLGN that likely corresponds to axons expressing only EGFP. In mice electroporated with EphA6/ephrinA5/EGFP $(n=14)$ or EphA6EE/ephrinA5/ EGFP $(n=9)$, the phenotypes were similar, with most axons projecting in the central dLGN (Fig. 7, Table 1). After electroporation with EphA6/ephrinA5 or EphA6EE/ephrinA5, we did not 
find axons in the VR areas. In general, less axons are observed in the dLGN than to the SC after electroporation at E13 (García-Frigola and Herrera, 2010), and this is probably the reason why axons were not detected in the VR areas of the dLGN when EphA6/ephrinA5 or EphA6EE/ephrinA5 are electroporated.

These results demonstrate that EphA6 is sufficient to direct axons to the dorsocaudal dLGN and that ephrinA5 expression in the RGCs is able to send axons to the VR geniculate areas independently of the signaling mediated by EphA6. EphA6 and ephrinA5 are downstream targets of Foxd1, and therefore these findings indicate that Foxd1 is essential for the correct establishment of retinogeniculate topographic maps.

\section{Discussion}

The experiments presented here solve the long-standing question of whether Foxd1 is required to specify temporal identity in the retina and demonstrate that this function is conserved in species with different visual system organizations. They also show that in the temporal retina, Foxd1 triggers the induction of EphA6 expression to direct axonal targeting to rostral collicular areas and represses ephinA5 expression to prevent axonal mistargeting in caudal areas. In addition, our experiments strongly support the hypothesis that the expression of ephrinA5 in the mammalian retina acts independently of EphA6 signaling, suggesting that the simple countergradient of EphA6 and ephrinA5 in the mouse retina is sufficient to establish the rostrocaudal topographic mapping in both the SC and the dLGN.

\section{Foxd1 nourishes the specification of ipsilateral RGCs}

In mammals, a proportion of axons arising in the temporal retina will project ipsilaterally, and therefore this retinal region sustains axonal ipsilaterality. We found that Foxd 1 is expressed in retinal progenitors in the temporal retina from very early stages and is downregulated before birth. The close spatiotemporal expression patterns of Foxd1 and Zic2 in the VT retina suggest that the expression of Foxd 1 in a restricted population of VT progenitors is determinant for the specification of iRGCs. Indeed, in the absence of Foxd1, the molecular program that specifies iRGCs (Zic2 and their targets, EphB1 and Sert) is missing, although Zic2 expression is not altered in the ciliary body. Also supporting this hypothesis, we show that when Foxd 1 is ectopically expressed in the embryonic retina, a number of central axons change laterality and avoid the midline. It is unlikely, however, that Foxd1 regulates Zic2 directly because Foxd 1 appears to be present in mature progenitors whereas Zic2 is expressed in early-differentiated iRGCs. In summary, our results demonstrate a function for Foxd1 in the specification of RGCs in the retina that is independent of its concomitant role in the patterning of the optic chiasm region.

\section{Foxd 1 is required for the expression of specific receptors essential for the proper establishment of topographic maps in the visual targets}

An essential feature of the visual temporal RGCs shared by mice and chickens is that temporal axons project to rostral areas in the $\mathrm{SC} /$ tectum. Foxd1 is expressed in the temporal retina at early stages in both chicks and mice. In the chick retina, the ectopic expression of Foxd1 is sufficient to send axons to the rostral tectum. However, until now it has remained unclear whether this protein is also required for the establishment of retinotopic maps. Our findings demonstrate that Foxd1 is necessary for the expression of EphAs in the temporal retina and for correct targeting in the collicular areas. Despite the conserved function of Foxd1 in retinotopy, different genetic programs are triggered in the two species. In mice, Foxd 1 is required to induce the program that specifies ipsilaterality, whereas in chicks this program is not expressed. In the chicken retina, the transcription factors SoHO and GH6 define a Foxd1 downstream program (Takahashi et al., $2003,2009)$ that does not seem to be conserved in the mouse.

The necessity to repress ephrinA5 in the temporal retina to prevent mistargeting to caudal collicular regions appears to be a feature common to both species. Our results open, however, the possibility that there are also differences in the mode of action of this guidance molecule between mice and birds. The expression of EphAs in the temporal retina of chick is homogenous, and ephrinA has been proposed as a generator of a gradient for EphAs activity (Carvalho et al., 2006). In mice, EphA6 and EphA5 are both expressed in a gradient in the temporal retina, making the desensitization of EphA receptors a superfluous mechanism. Therefore, it is more likely that in the mammalian retina, ephrinA 5 acts as a receptor rather than as a modulator of the EphAmediated signaling. The results presented here strongly reinforce this hypothesis. First, ectopic expression of ephrinA5 in the center of the retina leads to mistargeting of retinal axons to the caudal colliculus. EphrinA5 ectopically expressed in central retina cannot act through EphA5/6 signaling, because these receptors are expressed at very basal levels in central retina. Second, when ephrinA5 is overexpressed together with a constitutively active form of EphA6, axons are still mistargeted to the caudal collicular region.

Our ex vivo experiments show that Foxd1 is essential for proper targeting of RGC axons. The retinas that we used in these experiments were obtained at E15, before the establishment of topography in vivo; these experiments last $12 \mathrm{~d}$, and by the end of the experiment, these retinas are around similar stages as when axons normally establish retinotopic maps in the SC in vivo. Previous characterization of this model showed that axons from temporal wild-type retinas initially invade the entire SC to finally retract and project in more rostral areas (Nicol et al., 2006). Therefore, our coculture assay nicely recapitulates the formation of topographic maps along the rostrocaudal axis in the SC of wild-type mice. In the same time window, however, temporal axons from Foxd 1 mutants reached the end of the colliculus but failed to retract. EphA6, EphA5, ephrinA5, and Sert are all molecules, downstream of Foxd1, that are implicated in the formation of retinotopic maps (Drescher et al., 1995; Upton et al., 1999; Feldheim et al., 2000). Thus, although we cannot rule out that loss of Foxd 1 delays the formation of topographic maps, the simplest and most likely explanation to our ex vivo results is that Foxd1 is required for mapping in the SC and dLGN.

In conclusion, the experiments presented here demonstrate that the function of Foxd1 as a specifier of retinal temporal identity is conserved, but they also show that the downstream effectors triggered by this gene, and possibly their mechanisms of action, differ in birds and mammals.

\section{References}

Bardelli A, Longati P, Gramaglia D, Basilico C, Tamagnone L, Giordano S, Ballinari D, Michieli P, Comoglio PM (1998) Uncoupling signal transducers from oncogenic MET mutants abrogates cell transformation and inhibits invasive growth. Proc Natl Acad Sci U S A 95:14379-14383.

Brown A, Yates PA, Burrola P, Ortuno D, Vaidya A, Jessell TM, Pfaff SL, O’Leary DD, Lemke G (2000) Topographic mapping from the retina to the midbrain is controlled by relative but not absolute levels of EphA receptor signaling. Cell 102:77-88.

Brown LY, Kottman AH, Brown S (2003) Immunolocalization of zic2 expression in the developing forebrain. Gene Expr Patterns 3:361-367. 
Carvalho RF, Beutler M, Marler KJ, Knoll B, Becker-Barroso E, Heintzmann R, Ng T, Drescher U (2006) Silencing of EphA3 through a cis interaction with ephrinA5. Nat Neurosci 9:322-330.

Chen LI, Webster MK, Meyer AN, Donoghue DJ (1997) Transmembrane domain sequence requirements for activation of the p185c-neu receptor tyrosine kinase. J Cell Biol 137:619-631.

Cheng HJ, Nakamoto M, Bergemann AD, Flanagan JG (1995) Complementary gradients in expression and binding of ELF-1 and Mek4 in development of the topographic retinotectal projection map. Cell 82:371-381.

Connor RJ, Menzel P, Pasquale EB (1998) Expression and tyrosine phosphorylation of Eph receptors suggest multiple mechanisms in patterning of the visual system. Dev Biol 193:21-35.

Drescher U, Kremoser C, Handwerker C, Loschinger J, Noda M, Bonhoeffer F (1995) In vitro guidance of retinal ganglion cell axons by RAGS, a 25 $\mathrm{kDa}$ tectal protein related to ligands for Eph receptor tyrosine kinases. Cell 82:359-370.

Dütting D, Handwerker C, Drescher U (1999) Topographic targeting and pathfinding errors of retinal axons following overexpression of ephrinA ligands on retinal ganglion cell axons. Dev Biol 216:297-311.

Egea J, Nissen UV, Dufour A, Sahin M, Greer P, Kullander K, Mrsic-Flogel TD, Greenberg ME, Kiehn O, Vanderhaeghen P, Klein R (2005) Regulation of EphA 4 kinase activity is required for a subset of axon guidance decisions suggesting a key role for receptor clustering in Eph function. Neuron 47:515-528.

Feldheim DA, Vanderhaeghen P, Hansen MJ, Frisen J, Lu Q, Barbacid M, Flanagan JG (1998) Topographic guidance labels in a sensory projection to the forebrain. Neuron 21:1303-1313.

Feldheim DA, Kim YI, Bergemann AD, Frisen J, Barbacid M, Flanagan JG (2000) Genetic analysis of ephrin-A2 and ephrin-A5 shows their requirement in multiple aspects of retinocollicular mapping. Neuron 25:563-574.

Feldheim DA, Nakamoto M, Osterfield M, Gale NW, DeChiara TM, Rohatgi R, Yancopoulos GD, Flanagan JG (2004) Loss-of-function analysis of EphA receptors in retinotectal mapping. J Neurosci 24:2542-2550.

García-Frigola C, Herrera E (2010) Zic2 regulates the expression of Sert to modulate eye-specific refinement at the visual targets. EMBO J 29: $3170-3183$.

García-Frigola C, Carreres MI, Vegar C, Herrera E (2007) Gene delivery into mouse retinal ganglion cells by in utero electroporation. BMC Dev Biol 7:103.

García-Frigola C, Carreres MI, Vegar C, Mason C, Herrera E (2008) Zic2 promotes axonal divergence at the optic chiasm midline by EphB1dependent and -independent mechanisms. Development 135:1833-1841.

Guillery RW (1982) The optic chiasm of the vertebrate brain. Contrib Sensory Physiol 7:39-73.

Guillery RW, Mason CA, Taylor JSH (1995) Developmental determinants at the mammalian optic chiasm. J Neurosci 15:4727-4737.

Hatini V, Tao W, Lai E (1994) Expression of winged helix genes, BF-1 and BF-2, define adjacent domains within the developing forebrain and retina. J Neurobiol 25:1293-1309.

Hatini V, Huh SO, Herzlinger D, Soares VC, Lai E (1996) Essential role of stromal mesenchyme in kidney morphogenesis revealed by targeted disruption of Winged Helix transcription factor BF-2. Genes Dev 10: $1467-1478$

Herrera E, Brown LY, Aruga J, Rachel RA, Dolen G, Mikoshiba K, Brown S, Mason CA (2003) Zic2 patterns binocular vision by specifying the uncrossed retinal projection. Cell 114:545-557.

Herrera E, Marcus R, Li S, Williams SE, Erskine L, Lai E, Mason C (2004) Foxd1 is required for proper formation of the optic chiasm. Development 131:5727-5739.

Hornberger MR, Dutting D, Ciossek T, Yamada T, Handwerker C, Lang S, Weth F, Huf J, Wessel R, Logan C, Tanaka H, Drescher U (1999) Modulation of EphA receptor function by coexpressed ephrinA ligands on retinal ganglion cell axons. Neuron 22:731-742.

Huberman AD, Murray KD, Warland DK, Feldheim DA, Chapman B (2005)
Ephrin-As mediate targeting of eye-specific projections to the lateral geniculate nucleus. Nat Neurosci 8:1013-1021.

Marcus RC, Gale NW, Morrison ME, Mason CA, Yancopoulos GD (1996) Eph family receptors and their ligands distribute in opposing gradients in the developing mouse retina. Dev Biol 180:786-789.

Marler KJ, Becker-Barroso E, Martinez A, Llovera M, Wentzel C, Poopalasundaram S, Hindges R, Soriano E, Comella J, Drescher U (2008) A TrkB/EphrinA interaction controls retinal axon branching and synaptogenesis. J Neurosci 28:12700-12712.

Martin GR (2009) What is binocular vision for? A birds' eye view. J Vis 9:14 $11-19$.

Mason CA, Sretavan DW (1997) Glia, neurons, and axon pathfinding during optic chiasm development. Curr Opin Neurobiol 7:647-653.

Monschau B, Kremoser C, Ohta K, Tanaka H, Kaneko T, Yamada T, Handwerker C, Hornberger MR, Loschinger J, Pasquale EB, Siever DA, Verderame MF, Muller BK, Bonhoeffer F, Drescher U (1997) Shared and distinct functions of RAGS and ELF-1 in guiding retinal axons. EMBO J 16:1258-1267.

Nicol X, Muzerelle A, Rio JP, Metin C, Gaspar P (2006) Requirement of adenylate cyclase 1 for the ephrin-A5-dependent retraction of exuberant retinal axons. J Neurosci 26:862-872.

Nicol X, Voyatzis S, Muzerelle A, Narboux-Neme N, Sudhof TC, Miles R, Gaspar P (2007) cAMP oscillations and retinal activity are permissive for ephrin signaling during the establishment of the retinotopic map. Nat Neurosci 10:340-347.

Okabe M, Ikawa M, Kominami K, Nakanishi T, Nishimune Y (1997) "Green mice" as a source of ubiquitous green cells. FEBS Lett 407: 313-319.

O'Leary DD, Gerfen CR, Cowan WM (1983) The development and restriction of the ipsilateral retinofugal projection in the chick. Brain Res 312:93-109.

Pasini A, Geneste O, Legrand P, Schlumberger M, Rossel M, Fournier L, Rudkin BB, Schuffenecker I, Lenoir GM, Billaud M (1997) Oncogenic activation of RET by two distinct FMTC mutations affecting the tyrosine kinase domain. Oncogene 15:393-402.

Pfeiffenberger C, Cutforth T, Woods G, Yamada J, Renteria RC, Copenhagen DR, Flanagan JG, Feldheim DA (2005) Ephrin-As and neural activity are required for eye-specific patterning during retinogeniculate mapping. Nat Neurosci 8:1022-1027.

Rashid T, Upton AL, Blentic A, Ciossek T, Knoll B, Thompson ID, Drescher U (2005) Opposing gradients of ephrin-As and EphA7 in the superior colliculus are essential for topographic mapping in the mammalian visual system. Neuron 47:57-69.

Reber M, Burrola P, Lemke G (2004) A relative signalling model for the formation of a topographic neural map. Nature 431:847-853.

Schaeren-Wiemers N, Gerfin-Moser A (1993) A single protocol to detect transcripts of various types and expression levels in neural tissue and cultured cells: in situ hybridization using digoxigenin-labelled cRNA probes. Histochemistry 100:431-440.

Takahashi H, Shintani T, Sakuta H, Noda M (2003) CBF1 controls the retinotectal topographical map along the anteroposterior axis through multiple mechanisms. Development 130:5203-5215.

Takahashi H, Sakuta H, Shintani T, Noda M (2009) Functional mode of FoxD1/CBF2 for the establishment of temporal retinal specificity in the developing chick retina. Dev Biol 331:300-310.

Upton AL, Salichon N, Lebrand C, Ravary A, Blakely R, Seif I, Gaspar P (1999) Excess of serotonin (5-HT) alters the segregation of ispilateral and contralateral retinal projections in monoamine oxidase A knock-out mice: possible role of 5-HT uptake in retinal ganglion cells during development. J Neurosci 19:7007-7024.

Vanderhaeghen P, Lu Q, Prakash N, Frisen J, Walsh CA, Frostig RD, Flanagan JG (2000) A mapping label required for normal scale of body representation in the cortex. Nat Neurosci 3:358-365.

Yuasa J, Hirano S, Yamagata M, Noda M (1996) Visual projection map specified by topographic expression of transcription factors in the retina. Nature 382:632-635. 